\title{
Effects of Atorvastatin and Pravastatin on Malondialdehyde- Modified LDL in Hypercholesterolemic Patients
}

\author{
Akira Tamura, MD; Toru Watanabe, MD; Masaru Nasu, MD
}

\begin{abstract}
The aim of the present study was to compare the effects of atorvastatin and pravastatin on lipid parameters and the concentration of malondialdehyde-modified low-density lipoprotein (MDA-LDL) in hypercholesterolemic patients. A total of 17 patients (10 men, 7 women; mean age, $68 \pm 9$ years) who were indicated for drug therapy based on the National Cholesterol Education Program II underwent an 8-week regimen of atorvastatin $(10 \mathrm{mg} /$ day $)$ or pravastatin $(10 \mathrm{mg} /$ day $)$ with a 4 -week washout period between drugs. After an overnight fast, lipid parameters and MDA-LDL concentration were measured before and after the 8-week treatment with each drug. Both atorvastatin and pravastatin produced significant reductions in low-density lipoprotein (LDL) cholesterol and MDA-LDL concentrations, with a significant increase in high-density lipoprotein cholesterol concentration. The percent reductions in LDL cholesterol and MDA-LDL concentration were significantly greater with atorvastatin than pravastatin ( $46 \pm 6 \%$ vs $24 \pm 10 \%, \mathrm{p}<0.0001$, and $44 \pm 10 \%$ vs $14 \pm 13 \%, \mathrm{p}<0.0001$, respectively). The ratios of percent reductions in MDA-LDL concentrations and percent reductions in LDL cholesterol concentrations were significantly greater for atorvastatin than pravastatin $(0.96 \pm 0.19$ vs $0.59 \pm 0.55, \mathrm{p}<0.0001)$. In conclusion, atorvastatin reduced serum concentrations of LDL cholesterol and MDA-LDL to a greater degree than pravastatin, indicating that atorvastatin not only has stronger lipid-lowering effects, but also stronger antioxidative effects than pravastatin. (Circ J 2003; 67: 816-820)
\end{abstract}

Key Words: Hypercholesterolemia; Oxidized low-density lipoprotein; Statins

$\mathbf{O}$ xidized low-density lipoprotein (LDL) plays a key role in the initiation and development of atherosclerosis through a variety of effects, such as recruitment of monocytes, adhesion of leukocytes to endothelial cells, promotion of intraluminal thrombosis, activation of lymphocytes, enhanced cytotoxicity, and impairment of endothelium-dependent vasorelaxation! ${ }^{1-4}$ It has become possible to measure the serum concentrations of oxidized LDL or malondialdehyde-modified LDL (MDA-LDL) ${ }^{5-11}$ and their circulating concentrations have been reported to be significantly higher in patients with coronary artery disease (CAD) than in controls ${ }^{9-11}$ Sasaki et al reported that atorvastatin decreased the serum concentration of MDA-LDL in patients with mixed hyperlipoproteinemia ${ }^{12}$ and although atorvastatin produces greater reductions in the serum concentrations of total and LDL cholesterol than other statins, such as simvastatin, pravastatin, lovastatin, and fluvastatin, ${ }^{13}$ it is unclear whether this statin has more effect on decreasing the serum concentrations of MDA-LDL than other statins. In the present study, we compared the effects of atorvastatin and pravastatin on the serum concentrations of MDA-LDL in hypercholesterolemic patients.

\section{Methods}

\section{Study Population}

The study patients consisted of 17 patients (10 men, 7

(Received April 3, 2003; revised manuscript received June 26, 2003; accepted July 23, 2003)

Second Department of Internal Medicine, Oita Medical University, Oita, Japan

Mailing address: Akira Tamura, MD, Second Department of Internal Medicine, Oita Medical University, Hasama, Oita 879-5593, Japan.

E-mail: akira@oita-med.ac.jp women; mean age, $68 \pm 9$ years) who were indicated for drug therapy based on the National Cholesterol Education Program II ${ }^{4}$ Exclusion criteria were: (1) use of anti-inflammatory or lipid-lowering drugs or antioxidant supplements, (2) currently smoking, (3) renal or liver dysfunction, (4) diabetes mellitus, (5) the presence of angina attacks, (6) a history of stroke or acute coronary syndrome within 6 months, (7) a history of coronary interventional therapy within the past year, (8) chronic heart failure, (9) collagen disease, infection, malignant diseases, or recent $(<6$ months) major surgery or trauma, and (10) change in the drugs administered within the 3 months prior to registration in the study.

\section{Study Protocol}

The study protocol is shown in Fig 1. It was a randomized crossover design and the study started 1 month after registration. The patients underwent an 8-week regimen of statin therapy of either atorvastatin $(10 \mathrm{mg} /$ day $)$ or pravastatin $(10 \mathrm{mg} /$ day) with a 4-week washout period between drugs. The study protocol was in agreement with the guidelines of the institutional ethics committee and informed consent was obtained from each patient prior to enrollment in the study.

After an overnight fast, venous blood samples were obtained before and after the 8-week treatment with atorvastatin or pravastatin. The concentrations of LDL cholesterol, triglycerides, and HDL cholesterol were assayed by enzymatic methods. Serum concentrations of MDA-LDL were measured using an enzyme immunoassay kit (SRL, Inc, Tokyo, Japan). Intra- and inter-assay variations were within $3.5 \%$ and $6.9 \%$, respectively.

\section{Statistical Analysis}

All data are expressed as means \pm SD. Paired t test was 


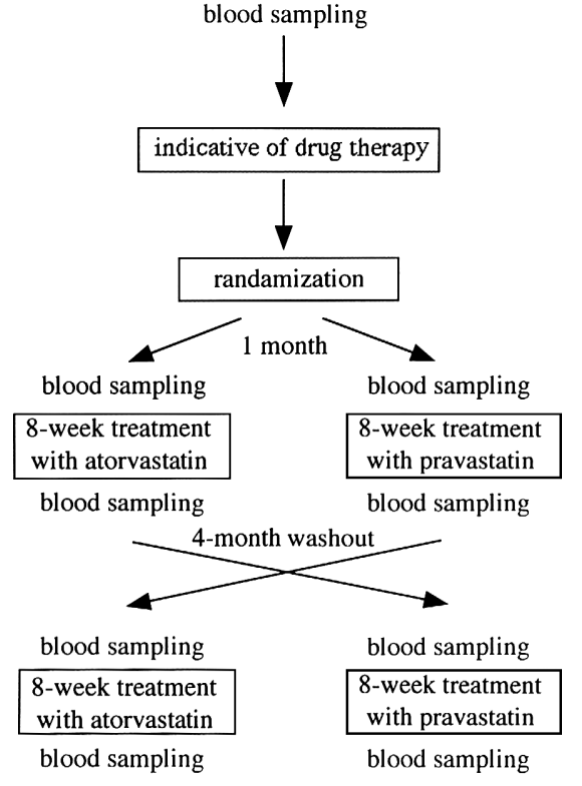

Fig 1. Study protocol.

Table 1 Patient Characteristics

\begin{tabular}{lc}
\hline \hline$n$ & 17 \\
Age, years & $68 \pm 9$ \\
Male (\%) & $10(59 \%)$ \\
Ex-smoker (\%) & $10(59 \%)$ \\
Hypertension (\%) & $13(76 \%)$ \\
Coronary artery disease (\%) & $15(88 \%)$ \\
Old cerebral infarction (\%) & $1(6 \%)$ \\
ACE inhibitors (\%) & $2(12 \%)$ \\
Calcium antagonists (\%) & $12(71 \%)$ \\
$\beta$-blockers (\%) & $4(24 \%)$ \\
Aspirin (\%) & $14(82 \%)$ \\
\hline
\end{tabular}

$A C E$, angiotensin-converting enzyme.

used to compare the differences between the data before and after the 8-week treatment with atorvastatin or pravastatin. The Pearson's correlation analysis was performed to estimate correlations between the concentrations of MDALDL and LDL cholesterol, triglyceride, or HDL cholesterol. A p value $<0.05$ was considered statistically significant.

\section{Results}

Patient characteristics are shown in Table 1. Of the 17 patients studied, there was a history of smoking and hypertension in $10(59 \%)$ and $13(76 \%)$, respectively. Fifteen patients (88\%) had CAD, but because of previous percutaneous transluminal coronary intervention, none of the patients experienced anginal attacks during the study. Angiotensin-converting enzyme inhibitors, calcium channel antagonists, $\beta$-blockers, and aspirin were currently being administered to $2(12 \%), 12$ (71\%), 4 (24\%), and 14 (82\%) patients, respectively.

Fig 2 shows the relationship between the serum concentrations of MDA-LDL and those of LDL cholesterol, triglycerides and HDL cholesterol. MDA-LDL tended to correlate positively with LDL cholesterol and triglycerides $(\mathrm{r}=0.43, \mathrm{p}=0.08$, and $\mathrm{r}=0.46, \mathrm{p}=0.06$, respectively). There was no significant correlation between the serum concentrations of MDA-LDL and HDL cholesterol $(\mathrm{r}=-0.15$,
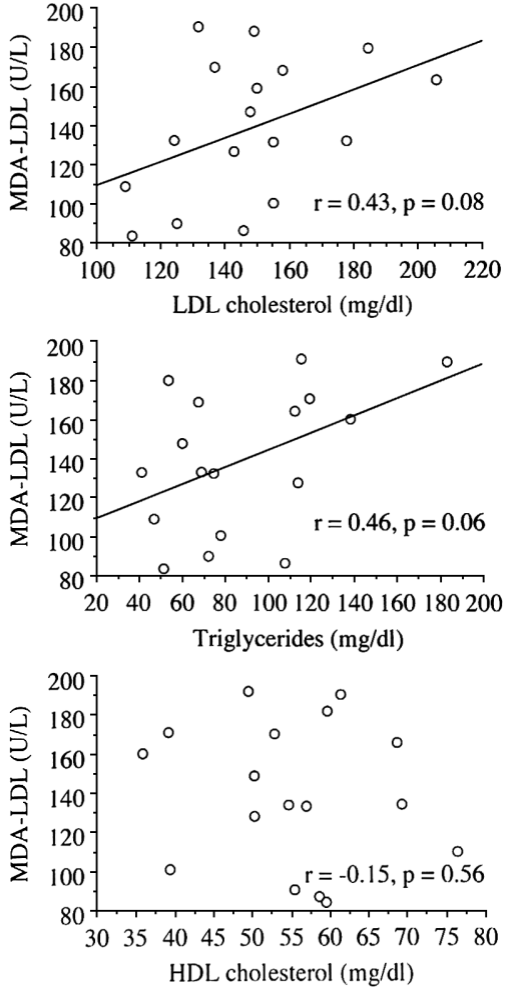

Fig 2. Relation of serum concentration of malondialdehyde-modified low-density lipoprotein (MDA-LDL) to those of LDL cholesterol, triglyceride, or HDL cholesterol.

$\mathrm{p}=0.56)$.

Table 2 shows the lipid parameters and MDA-LDL concentrations before and after the 8-week treatment with either atorvastatin or pravastatin. Both statins produced significant reductions in the serum concentrations of LDL cholesterol and MDA-LDL, with a significant increase in that of HDL cholesterol. Serum concentrations of triglycerides were significantly reduced only by atorvastatin. Fig 3 shows the percent reductions in lipid parameters and MDA-LDL concentrations by the 8-week treatments. Percent reductions in serum concentrations of LDL cholesterol and MDA-LDL were significantly greater with atorvastatin than pravastatin $(46 \pm 6 \%$ vs $24 \pm 10 \%, p<0.0001$ and $44 \pm$ $10 \%$ vs $14 \pm 13 \%, \mathrm{p}<0.0001$, respectively). The percent reductions in LDL cholesterol and MDA-LDL by pravastatin did not differ significantly between patients administered the drug initially and those who received it after the cross-over $(24 \pm 11 \%$ vs $24 \pm 11 \%$ and $14 \pm 11 \%$ vs $14 \pm 15 \%$, respectively) nor was there any significant difference for the patients who were given atorvastatin $(45 \pm 7.5 \%$ vs $47 \pm 4.5 \%$ (initial treatment) and $42 \pm 10 \%$ vs $46 \pm 11 \%$ (after cross-over), respectively). The ratios of percent reductions in MDA-LDL concentrations and percent reductions in LDL cholesterol concentrations were significantly greater for atorvastatin than pravastatin $(0.96 \pm 0.19$ vs $0.59 \pm 0.55$, $\mathrm{p}<0.0001$ ) (Fig 4). Percent reductions in triglyceride concentrations tended to be greater with atorvastatin than pravastatin $(26 \pm 21 \%$ vs $8.9 \pm 28 \%, \mathrm{p}=0.053)$. The percent increases in HDL cholesterol did not differ significantly between atorvastatin and pravastatin $(10 \pm 14 \%$ vs $9.8 \pm 14 \%)$.

No adverse effects were observed in any patients. 
Table 2 Changes in Serum Lipid Parameters and MDA-LDL Concentrations Before and After Treatment With Atorvastatin and Pravastatin

\begin{tabular}{|c|c|c|c|c|c|c|}
\hline & \multicolumn{2}{|c|}{ Atorvastatin } & \multirow{2}{*}{$p$ value } & \multicolumn{2}{|c|}{ Pravastatin } & \multirow{2}{*}{$p$ value } \\
\hline & Before & After & & Before & After & \\
\hline LDL cholesterol, $\mathrm{mg} / \mathrm{dl}$ & $150 \pm 26$ & $80 \pm 18$ & $<0.0001$ & $149 \pm 26$ & $112 \pm 17$ & $<0.0001$ \\
\hline$M D A-L D L, U / L$ & $157 \pm 49$ & $85 \pm 20$ & $<0.0001$ & $147 \pm 36$ & $123 \pm 27$ & 0.0015 \\
\hline Triglycerides, $\mathrm{mg} / \mathrm{dl}$ & $102 \pm 46$ & $69 \pm 24$ & 0.0007 & $84 \pm 31$ & $76 \pm 35$ & 0.15 \\
\hline HDL cholesterol, $\mathrm{mg} / \mathrm{dl}$ & $57 \pm 12$ & $63 \pm 12$ & 0.0017 & $58 \pm 13$ & $63 \pm 13$ & 0.11 \\
\hline
\end{tabular}

Data are presented as mean $\pm S D$. LDL, low-density lipoprotein; MDA, malondialdehyde-modified; HDL, high-density lipoprotein.

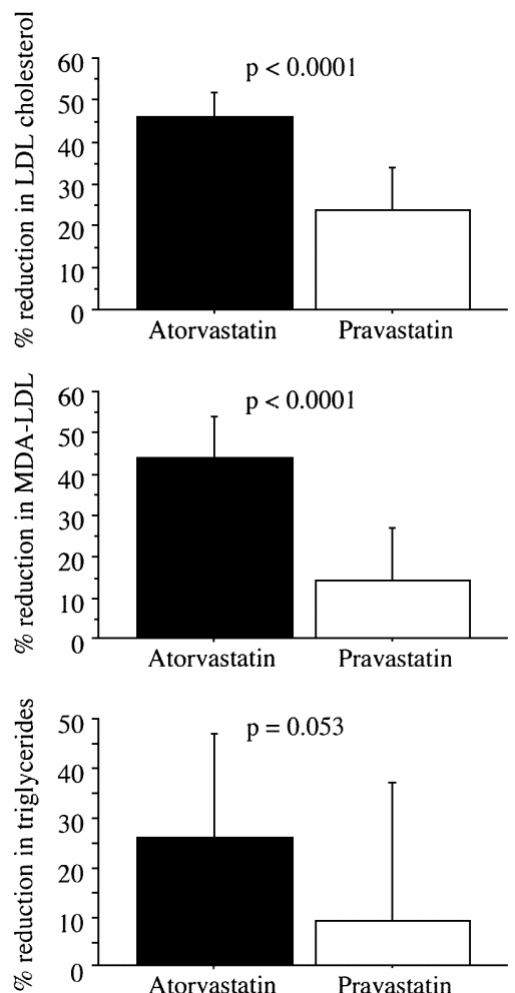

Fig 3. Percent reductions in serum concentrations of LDL cholesterol, MDA-LDL, and triglycerides after 8-week treatment with atorvastatin or pravastatin.

\section{Discussion}

In the present study, both atorvastatin and pravastatin decreased LDL cholesterol and MDA-LDL concentrations in hypercholesterolemic patients, but these effects were significantly greater with atorvastatin. These results indicate that atorvastatin not only has stronger lipid-lowering effects but also stronger antioxidant effects than pravastatin.

In hypercholesterolemic patients, the concentration of circulating LDL is increased, and the LDL is more aged and more susceptible to oxidative modifications than LDL from healthy subjects 15 Indeed, in the present study, serum MDA-LDL concentrations tended to correlate positively with those of LDL cholesterol. Therefore, the greater reduction in the serum MDA-LDL concentrations by atorvastatin might result from the greater reduction in serum LDL cholesterol. Because 'aged' LDL is more prone to oxidative processes during its long presence in the circulation, a substantial removal of 'aged' LDL via increased LDL receptor activity, mainly in the liver, could contribute to a

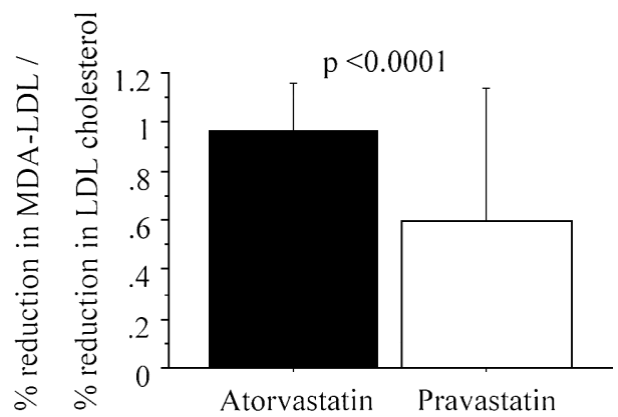

Fig 4. Ratios of percent reductions in serum concentration of MDALDL and percent reductions in LDL-cholesterol concentration after 8week treatment with atorvastatin or pravastatin.

reduction in the susceptibility of the 'new' LDL population in the circulation to oxidation, 16 leading to the reduced levels of circulating MDA-LDL. However, the ratios of percent reduction in MDA-LDL and LDL cholesterol were significantly greater for atorvastatin than pravastatin, which suggests that with atorvastatin other mechanisms independent of the LDL cholesterol-lowering effect contribute more to the reduction in serum MDA-LDL concentration. The para- and ortho-hydroxymetabolites of atorvastatin, but not the parent compound, possess antioxidative properties $^{17}$ and the protective effect of these hydroxymetabolites against the oxidation of LDL may have led to the greater decrease in MDA-LDL.

Statins have been shown to exert antioxidant effects by reducing the expression of essential $\mathrm{NAD}(\mathrm{P}) \mathrm{H}$ oxidase subunits that are the predominant source of reactive oxygen species in the vessel wall. ${ }^{18}$ However, it is unclear whether this effect contributes to the reduction in circulating MDALDL concentrations.

Endothelial dysfunction is an initiating factor in the development of atherosclerosis ${ }^{19}$ and oxidized LDL evokes endothelial dysfunction by impairing the signal transduction between endothelial cell surface receptors and nitric oxide production ${ }^{20}$ inhibiting nitric oxide synthase expression, 21,22 increasing the oxidative degradation of nitric oxide, 23 and inducing apoptosis ${ }^{24-26}$ Oxidized LDL also disturbs vasomotion by direct interaction with vascular smooth muscle cells 27 We previously reported that there was a significant correlation between the plasma concentrations of oxidized LDL and the coronary vasomotor response to acetylcholine in patients with stable $\mathrm{CAD}^{28}$ which suggested that the circulating oxidized LDL concentration may indirectly reflect the impairment of endothelium-dependent vasodilation. Zhu et al demonstrated that atorvastatin reduces the concentration of lysophosphatidylcholine in oxidized LDLs, leading to improved endothelium-depen- 
dent relaxation of blood vessels ${ }^{29}$ Given recent observations that endothelial dysfunction predicts future atherosclerotic disease and cardiovascular events, ${ }^{30,31}$ a reduction in the serum concentration of MDA-LDL which is a major component of oxidized LDLs, may reduce the likelihood of future atherosclerotic disease and cardiovascular events. Indeed, a recent study reported that the concentration of circulating oxidized LDL is an important predictor for cardiac events in patients with $\mathrm{CAD}^{32}$ Further studies are needed to clarify whether, because of its greater lipidlowering and antioxidative effects, atorvastatin is the more advantageous than other statins in the management of hypercholesterolemic patients.

\section{Study Limitations}

First, the number of patients studied was small and therefore, large population studies are needed to confirm our results. Second, the frequency of patients with CAD was extremely high and therefore, our results cannot be generalized to all patients with hypercholesterolemia. The status of CAD could affect the serum concentration of MDA-LDL; Holvoet et al have shown that plasma concentrations of MDA-LDL were significantly higher in patients with acute coronary syndrome? However, in the present study, none of the patients had experienced acute coronary syndrome within the 6 months prior to the study. Third, 13 $(76 \%)$ of the present patients were being treated with angiotensin-converting enzyme inhibitors and/or calcium channel antagonists, which could affect the concentration of MDA-LDL33,34 However, these treatments were started at least 3 months prior to registration, and their dosages were not changed within 3 months of registration or during the study. Therefore, we do not believe that these drugs strongly affected our results.

\section{Conclusions}

Atorvastatin reduced the serum concentrations of LDL cholesterol and MDA-LDL to a greater degree than pravastatin, indicating that it not only has stronger lipidlowering effects but also stronger antioxidative effects than pravastatin. Further studies will be needed to clarify whether atorvastatin is the more advantageous drug for the management of hypercholesterolemic patients.

\section{References}

1. Steinberg D, Parthasarathy S, Carew TE, Khoo JC, Witztum JL. Beyond cholesterol: Modifications of low-density lipoprotein that increase its atherogenicity. N Engl J Med 1989; 320: 915-924.

2. Witztum JL, Steinberg D. Role of oxidized low density lipoprotein in atherogenesis. J Clin Invest 1991; 88: 1785-1792.

3. Steinberg D. Role of oxidized LDL and antioxidants in atherosclerosis. Adv Exp Med Biol 1995; 369: 39-48.

4. Witztum JL, Steinberg D. The oxidative modification hypothesis of atherosclerosis: Does it hold for humans? Trends Cardiovasc Med 2001; 11: 93-102.

5. Kotani K, Maekawa M, Kanno T, Kondo A, Toda N, Manabe M. Distribution of immunoreactive malondialdehyde-modified low-density lipoprotein in human serum. Biochim Biophys Acta 1994; 1215: $121-125$.

6. Itabe H, Yamamoto H, Imanaka T, Shimamura K, Uchiyama H, Kimura J, et al. Sensitive detection of oxidatively modified low density lipoprotein using a monoclonal antibody. J Lipid Res 1996; 37: $45-53$.

7. Palinski W, Horkko S, Miller E, Steinbrecher UP, Powell HC, Curtiss LK, et al. Cloning of monoclonal autoantibodies to epitopes of oxidized lipoproteins from apolipoprotein E-deficient mice: Demonstration of epitopes of oxidized low density lipoprotein in human plasma. J Clin Invest 1996; 98: 800-814.

8. Holvoet P, Stassen JM, Van Cleemput J, Collen D, Vanhaecke J. Oxidized low density lipoproteins in patients with transplant-associated coronary artery disease. Arterioscler Thromb Vasc Biol 1998; 18: $100-107$.

9. Holvoet P, Vanhaecke J, Janssens S, Van de Werf F, Collen D. Oxidized LDL and malondialdehyde-modified LDL in patients with acute coronary syndromes and stable coronary artery disease. Circulation 1998; 98: 1487-1494.

10. Toshima S, Hasegawa A, Kurabayashi M, Itabe H, Takano T, Sugano J, et al. Circulating oxidized low density lipoprotein levels: A biochemical risk marker for coronary heart disease. Arterioscler Thromb Vasc Biol 2000; 20: 2243-2247.

11. Tanaga K, Bujo H, Inoue M, Mikami K, Kotani K, Takahashi K, et al. Increased circulating molandialdehyde-modified LDL levels in patients with coronary artery diseases and their association with peak sizes of LDL particles. Arterioscler Thromb Vasc Biol 2002; 22: $662-666$.

12. Sasaki S, Kuwahara N, Kunitomo K, Harada S, Yamada T, Azuma $\mathrm{A}$, et al. Effects of atorvastatin on oxidized low-density lipoprotein, low-density lipoprotein subfraction distribution, and remnant lipoprotein in patients with mixed hyperlipoproteinemia. Am J Cardiol 2002; 89: 386-389.

13. Jones P, Kafonek S, Laurora I, Hunninghake D, for the CURVES investigators. Comparative dose efficacy study of atorvastatin versus simvastatin, pravastatin, lovastatin, and fluvastatin in patients with hypercholesterolemia (The CURVES study). Am J Cardiol 1998; 81: $582-587$.

14. National Cholesterol Education Program: Second report of the expert panel on detection, evaluation, and treatment of high blood cholesterol in adults (Adult Treatment Panel II). Circulation 1994; 89: $1333-$ 1445 .

15. Lavy A, Brook GJ, Dankner G, Amotz AB, Aviram M. Enhanced in vitro oxidation of plasma lipoproteins derived from hypercholesterolemic patients. Metabolism 1991; 40: 794-799.

16. Hussein O, Schlezinger S, Rosenblat M, Keidar S, Aviram M. Reduced susceptibility of low density lipoprotein (LDL) to lipid peroxidation after fluvastatin therapy is associated with the hypocholesterolemic effect of the drug and its binding to the LDL. Atherosclerosis 1997; 128: 11-18.

17. Aviram M, Rosenblat M, Bisgaier CL, Newton RS. Atorvastatin and gemfibrozil metabolites, but not the parent drugs, are potent antioxidants against lipoprotein oxidation. Atherosclerosis 1998; 138: $271-280$.

18. Wassmann S, Laufs U, Müller K, Konkol C, Ahlbory K, Bäumer AT, et al. Cellular antioxidant effects of atorvastatin in vitro and in vivo. Arterioscler Thromb Vasc Biol 2002; 22: 300-305.

19. Ross R. Atherosclerosis: An inflammatory disease. $N$ Engl J Med 1988; 319: $512-513$

20. Kugiyama K, Kerns SA, Morrisett JD, Roberts R, Henry PD. Impairment of endothelium-dependent arterial relaxation by lysolecithin in modified low-density lipoproteins. Nature 1990; 344: 160-162.

21. Yang X, Cai B, Sciacca RR, Cannon PJ. Inhibition of inducible nitric oxide synthase in macrophages by oxidized low-density lipoproteins. Circ Res 1994; 74: 318-328.

22. Liao JK, Shin WS, Lee WY, Clark SL. Oxidized low-density lipoprotein decreases the expression of endothelial nitric oxide synthase. J Biol Chem 1995; 270: 319-324.

23. Ohara Y, Peterson TE, Harrison DG. Hypercholesterolemia increases endothelial superoxide anion production. J Clin Invest 1993; 91: $2546-2551$.

24. Harada-Shiba M, Kinoshita M, Kamido H, Shimokado K. Oxidized low density lipoprotein induced apoptosis in cultured human umbilical vein endothelial cells by common and unique mechanisms. $J$ Biol Chem 1998; 273: $9681-9687$.

25. Dimmeler S, Haendeler J, Galle J, Zeiher AM. Oxidized low-density lipoprotein induces apoptosis of human endothelial cells by activation of CPP32-like protease: A mechanistic clue to the 'response to injury' hypothesis. Circulation 1997; 95: 1760-1763.

26. Imanishi T, Hano T, Sawamura T, Takarada S, Nishio I. Oxidized low density lipoprotein potentiation of Fas-induced apoptosis through lectin-like oxidized-low density lipoprotein receptor-1 in human umbilical vascular endothelial cells. Circ J 2002; 66: 1060-1064.

27. Galle J, Bassenge E, Busse R. Oxidized low density lipoproteins potentiate vasoconstrictions to various agonists by direct interaction with vascular smooth muscle. Circ Res 1990; 66: 1287-1293.

28. Nakaishi T, Tamura A, Watanabe T, Mikuriya Y, Nasu M. Relationship between plasma oxidized low-density lipoprotein and the coronary vasomotor response to acetylcholine in patients with coronary artery disease. Jpn Circ J 2000; 64: 856-860. 
29. Zhu Q, McMaster J, Mymin D, Dembinski T, Hatch G, Choy PC, et al. Effects of atorvastatin treatment on the oxidatively modified low density lipoprotein in hyperlipidemic patients. Mol Cell Biochem 2000; 207: 9-17.

30. Suwaidi JA, Hamasaki S, Higano ST, Nishimura RA, Holmes DR Jr, Lerman A. Long-term follow-up of patienst with mild coronary artery disease and endothelial dysfunction. Circulation 2000; 101: $948-954$.

31. Schächinger V, Britten MB, Zeiher AM. Prognostic impact of coronary vasodilator dysfunction on adverse long-term outcome of coronary heart disease. Circulation 2000; 101: 1899-1906.
32. Shimada K, Mokuno H, Matsunaga E, Yamashita H, Satoh Y, Miyazaki T, et al. Circulating oxidized LDL is an important predictor for cardiac events in patients with coronary artery disease (abstract). Circ J 2003; 67(Suppl I): 447.

33. Bagchi D, Prasad R, Das DK. Direct scavenging of free radicals by captopril, an angiotensin converting enzyme inhibitor. Biochem Biophys Res Commun 1989; 158: 52-57.

34. Janero DR, Burghardt B, Lopez R. Protection of cardiac membrane phospholipid against oxidative injury by calcium antagonists. Biochem Pharmacol 1988; 37: 4197-4203. 